\title{
A predictable smoothing evolution model for computer-controlled polishing
}

\author{
Jing Hou', Pengli Lei ${ }^{1}$, Shiwei Liu ${ }^{2 *} \mathbb{D}$, Xianhua Chen ${ }^{1}$, Jian Wang ${ }^{1}$, Wenhui Deng ${ }^{1}$ and Bo Zhong ${ }^{1}$
}

\begin{abstract}
Quantitative prediction of the smoothing of mid-spatial frequency errors (MSFE) is urgently needed to realize process guidance for computer controlled optical surfacing (CCOS) rather than a qualitative analysis of the processing results. Consequently, a predictable time-dependent model combining process parameters and an error decreasing factor (EDF) were presented in this paper. The basic smoothing theory, solution method and modification of this model were expounded separately and verified by experiments. The experimental results show that the theoretical predicted curve agrees well with the actual smoothing effect. The smoothing evolution model provides certain theoretical support and guidance for the quantitative prediction and parameter selection of the smoothing of MSFE.
\end{abstract}

Keywords: Mid-spatial frequency errors, Computer-controlled polishing, Smoothing, Quantitative prediction, Parametric

\section{Introduction}

In the past few decades, computer controlled optical surfacing (CCOS) has been widely and successfully applied to the manufacture of optical components [1-3], providing a deterministic material removal technology for optical devices [4], such as small-sized optical lenses, large astronomical telescopes and high-power laser systems. Different processing methods are commonly used, which has a broad coverage including CNC polishing, gasbag polishing, magnetorheological polishing, ion beam polishing, etc [5-7] In some extreme optical systems like large-aperture telescope systems or nanoscale lithography systems, surface errors of the optical components play a critical role in the imaging and operation quality of the entire system. In consequences, study on the formation mechanism and suppression method of surface errors is of great significance to the process and manufacturing.

The surface errors of optical components can be classified as low-spatial frequency errors, mid-spatial frequency

\footnotetext{
* Correspondence: liushiwei0916@163.com

${ }^{2}$ School of Mechatronics \& Engineering, Harbin Institute of Technology, Harbin 150001, China

Full list of author information is available at the end of the article
}

errors (MSFE) and high-spatial frequency errors, according to the spatial frequency. The low-spatial frequency error is a shape error, which can introduce various aberrations and lead to image distortion of the optical system; the mid-spatial frequency error represents the ripples of the component surface, which can result in the small angle scattering of light and affect the imaging contrast; the high-spatial frequency error represents the roughness of the surface which enables large-angle scattering of light and reduces specular reflectance. Therefore, sub-band research and surface error control play a key role in the processing and evaluation of optical systems.

In recent years, lots of research have been done on the smoothing of the surface errors. In 1981, Brown and Parks quantitatively explained the smoothing effect with elastic support flexible abrasive belts [8]. Mehta and Reid first proposed flexible pads in 1990 and built bridge models based on elastic theory $[9,10]$. After 2010, Kim did a lot of research work on RC pads. A parametric mathematical model was proposed based on the bridge model to describe the polishing effect and efficiency of various polishing processes $[11,12]$. Later on, Y. Shu pointed out that Kim's model gave a flat uniform slip factor $(S F)$, by ignoring the time-varying characteristics. 
When considering the smoothing time during the polishing process, the evolution of surface errors was revealed and an exponentially decreasing function image was obtained [13]. And by comparing the different polishing pad movements, it was pointed out that the smoothing curve under the double planetary motion drops faster and the smoothing limit value is smaller, indicating that the double planetary motion polishing pad has better smoothing effect. At the same time, the addition of random polishing paths in the smoothing process also achieved progress $[14,15]$. Nie analyzed the smoothing effect of irregular ripples with finite element method [16].

With some of the theoretical models mentioned above, the effects of different process parameters on the smoothing process have also been studied. Zhang compared the smoothing experiment of the asphalt disk with the polyurethane pad at the same speed, finding that the pitch pad had better smoothing effect than the polyurethane pad, and higher speed resulted in higher smoothing efficiency [17]. With the parametric model, Kim compared the smoothing factors of different polishing tools, finding that the elastic material with harder surface had better smoothing effect [18]. Nie found that the polishing pad groove did affect the material removal and also the radial slotting method had a better smoothing effect by changing the slotting method of the polishing pad groove [19].

In this paper, the smoothing mechanism of the midspatial frequency errors in computer-controlled polishing using pitch pads was studied. Based on the existing theoretical model, a further extension and derivation of the smoothing theory has been carried out. Considering the actual polishing process, a predictable smoothing evolution model was established. It is promising that this model can provide more precise guidance and prediction for the actual smoothing process of the surface ripple errors.

\section{Method}

\section{Smoothing theory}

It is very important to establish a reasonable and effective mathematical model of the smoothing effect in the computer-controlled polishing process. There have been some studies on the smoothing effect by using elastic tools mentioned above.

As shown in Fig. 1(a), the ripple errors on the surface cause uneven contact between the polishing pad and the workpiece, resulting in inhomogeneity in the pressure distribution. The peak of the ripple has an additional pressure difference $P_{\text {add }}$ compared to that of the trough. According to the Preston equation, the material removed during the polishing process is proportional to the polishing pressure. Therefore, more material is removed at the peak of the ripple than at the trough. As a result, the workpiece will become smoother, thereby achieving the smoothing effect of the surface errors. The Preston equation shows that the material removal of workpiece satisfies:

$$
\Delta \varepsilon=K \cdot P_{a d d} \cdot v \cdot \Delta t
$$

where $\Delta \varepsilon$ is the amount of change in the amplitude of the ripple errors after single smoothing, $K$ is a constant parameter, $v$ is the relative speed between the polishing pad and the workpiece, and $\Delta t$ is the time of single smoothing based on the existing surface errors.

For visco-elastic polishing tools, such as pitch pads or RC pads, a parametric smoothing model [11] has been established:

$$
S F=\frac{\Delta \varepsilon}{\Delta Z}=k \cdot\left(\varepsilon_{\text {ini }}-\varepsilon_{0}\right)
$$

where $S F$ is the smoothing factor, defined as the ratio of $\Delta \varepsilon$ (the amount of change in the amplitude of the ripple errors after single smoothing) to $\Delta Z$ (the depth of material removal on the surface of the workpiece after single smoothing under the premise that the contact pressure at the peak and the trough of the ripple errors is the same). To a certain extent, $\Delta Z$ can be understood as the depth of material removal on the surface of the workpiece without ripple errors under the same processing conditions. $\varepsilon_{i n i}$ is the initial amplitude of the ripple errors before smoothing and $\varepsilon_{0}$ is the final amplitude after multiple smoothing which indicates the limit of the smoothing process, that is, the amplitude does not change with the increase of smoothing time when the amplitude of ripple errors decreases to $\varepsilon_{0}$. The magnitude of $S F$ characterizes the smoothing capacity to the ripple errors in the polishing process. It can be seen that the smoothing factor $S F$ has a linear correlation with the surface roughness of the workpiece, and the proportional coefficient is $k$. In the parametric smoothing model:

$$
\begin{aligned}
& k=\frac{\kappa_{\text {total }}}{P} \\
& \frac{1}{\kappa_{\text {total }}}=\frac{1}{\kappa_{\text {elastic }}}+\frac{1}{\kappa_{\text {others }}}
\end{aligned}
$$

where $P$ is the pressure between the polishing pad and the workpiece, $\kappa_{\text {total }}$ is the material coefficient of the polishing pad, which is related to the elastic material coefficient $\kappa_{\text {elastic }}$ and the overall material coefficient $\kappa_{\text {others }}$ of other structures.

The parametric smoothing model indicates that the smoothing factor is related to the material parameters and polishing pressure during the polishing process. 


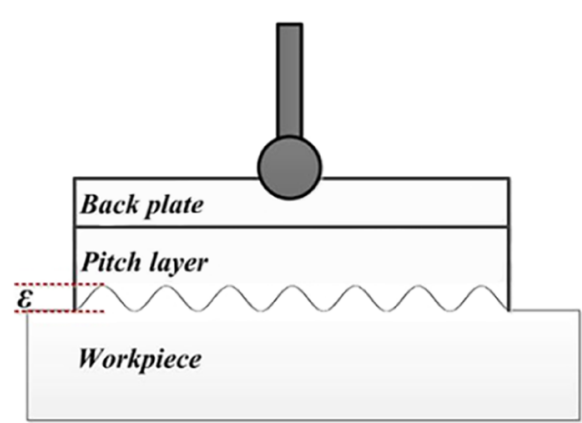

(a)

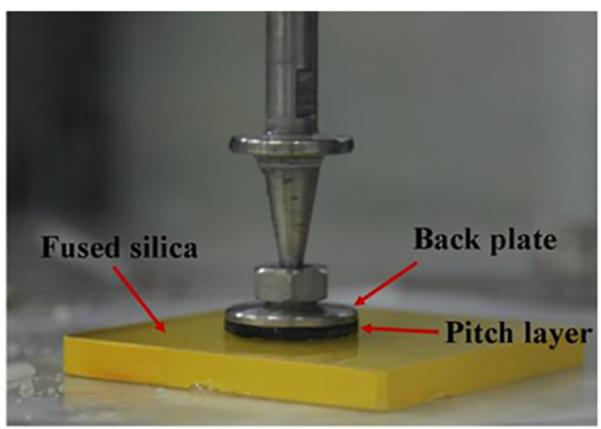

(b)

Fig. 1 a A pitch polishing pad contact on the workpiece with ripple errors. b) Experiment platform

However, it is difficult to reflect the relationship between the parameters of smoothing process and other process. It is not intuitive to infer the material removal rate of the actual polishing process because no factor is included in this model to describe the evolution of the surface error with time. Therefore, based on the parametric model, a time-dependent smoothing evolution model has also been proposed and applied [13].

Using the mathematical expression of the smoothing factor in the parametric smoothing model, the following equation can be obtained:

$$
\frac{d \varepsilon}{d Z}=\frac{d\left(\varepsilon-\varepsilon_{0}\right)}{d Z}=-k \cdot\left(\varepsilon-\varepsilon_{0}\right)
$$

Then

$$
\varepsilon=\left(\varepsilon_{i n i}-\varepsilon_{0}\right) \cdot e^{-k \cdot Z}+\varepsilon_{0}
$$

where $\varepsilon$ is the amplitude of the ripple errors on the workpiece surface after several times of smoothing process, $Z$ is the total material removal depth of the workpiece surface without ripple errors under the same smoothing time $t$ and processing conditions.

With $Z$ developed by the Preston equation, similar as Eq.1, combined with Eq.3, the above equation yields:

$$
\varepsilon=\left(\varepsilon_{\text {ini }}-\varepsilon_{0}\right) \cdot e^{-\kappa_{\text {total }} \cdot K \cdot v \cdot t}+\varepsilon_{0}
$$

The smoothing model represented by Eq.7 reveals that the surface ripple errors converge exponentially with time during the smoothing process. In the general application of the model, the data points of the polishing process are often fitted by an exponential function, and the smoothing efficiency is measured by the obtained fitting parameter values. However, how to calculate a more accurate predicted curve of smoothing process from the various process parameters of the model is still an urgent problem to be solved. The theoretical model established next is to find the relationship between the practical polishing parameters and the smoothing efficiency by analyzing the actual smoothing parameters in computer-controlled polishing.

\section{Predictable evolution smoothing model}

Starting from Eq.6, the material removal depth $Z$ under different processing conditions can be obtained by relatively accurately modeling and simulating of the theoretical tool influence function (TIF). In computercontrolled polishing, the polishing pad usually moves in a specific mode. The most common mode for pitch pad is double planetary motion, as shown in Fig. 2.

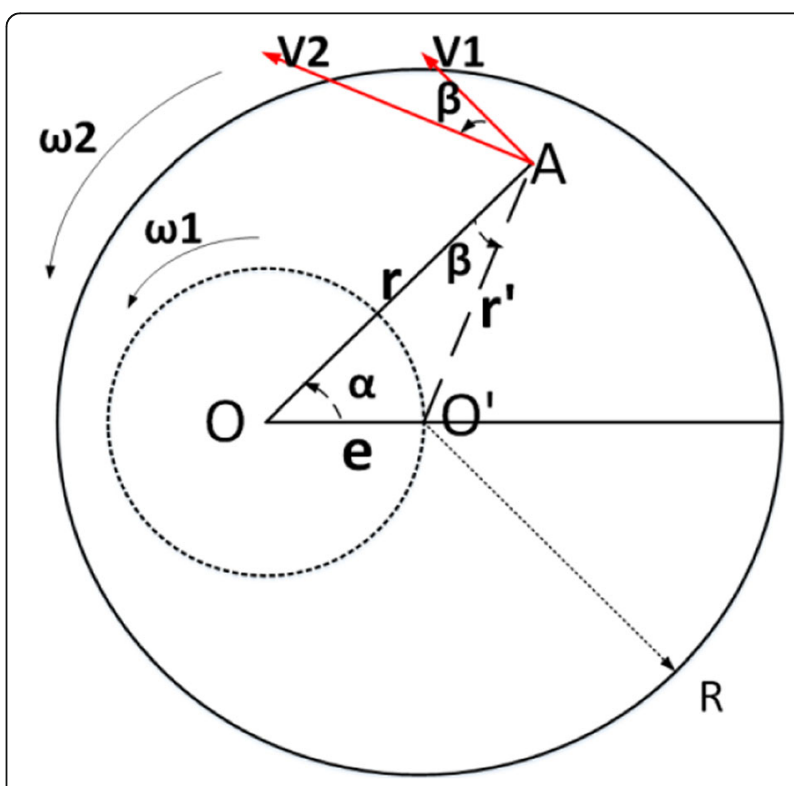

Fig. 2 The double planetary motion of the polishing pad, where $R$ is the radius of the polishing pad. The polishing pad has a revolution $\omega_{1}$ around a certain circle $O$ at a certain eccentricity $e$, and a rotation with angular velocity $\omega_{2}$. The distance between the polishing point $A$ and the center $O$ is $r$. Since the polishing pad motion has both revolution and rotation, with the corresponding linear velocities being $v_{1}$ and $v_{2}$, respectively, the velocity $v$ of the polishing pad relative to the workpiece is the vector sum of $v_{1}$ and $v_{2}$. The angle between $v_{7}$ and $v_{2}$ is $\beta$. The angle between $O, A$ and $O^{\prime}$ is $a$ 
The polishing velocity $v$ of any contact point $(r, \alpha)$ on the polishing pad surface varies with the locations, which can be expressed as:

$$
v(r, \alpha)=\omega_{1} \sqrt{r^{2}(1+n)^{2}+e^{2} n^{2}-2 \operatorname{ren}(1+n) \cos \alpha}
$$

where

$$
n=\frac{\omega_{2}}{\omega_{1}}
$$

Under a uniform pressure distribution of the polishing layer, the TIF of the double planetary motion pad (the average removal within a period $T$ ) satisfies:

$$
\begin{aligned}
T I F & =K \cdot P \cdot v=K \cdot P \cdot \frac{\int_{-\theta}^{\theta} v(r, \alpha) d \alpha}{T} \\
& =K \cdot P \cdot \frac{\omega_{1}}{2 \pi} \int_{-\theta}^{\theta} v(r, \alpha) d \alpha
\end{aligned}
$$

where the integration interval $\theta$ satisfies the following condition:

$$
\theta=\left\{\begin{array}{cc}
r & \leq R-e \\
2 \arccos \left(\frac{r^{2}+e^{2}-R^{2}}{2 r e}\right) & R-e<r \leq R+e \\
0 & r>R+e
\end{array}\right.
$$

The TIF is related to the position $r$ as can be seen from the above equation. Meanwhile, in the polishing process, the area of a 2-D TIF image contains all the points at which the polishing pad can produce material removal. Therefore, an overall analysis of the TIF area is carried out to establish a comprehensive average effect of material removal. Then the total volume removal rate $(V R R)$ in the area of the tool influence function satisfies:

$$
\begin{aligned}
V R R & =\iint T I F(x, y) d x d y=K \cdot P \cdot \frac{\omega_{1}}{2 \pi} \int_{0}^{2 \pi} d \phi \int_{0}^{R} r V(r) d r \\
& =K \cdot P \cdot \omega_{1} \int_{0}^{R} r V(r) d r
\end{aligned}
$$

where

$$
\begin{aligned}
V(r) & =\frac{1}{\omega_{1}} \int_{-\theta}^{\theta} v(r, \alpha) d \alpha \\
& =\int_{-\theta}^{\theta} \sqrt{r^{2}(1+n)^{2}+e^{2} n^{2}-2 \operatorname{ren}(1+n) \cos \alpha} d \alpha
\end{aligned}
$$

During the actual polishing process, due to the non-uniformity of the velocity distribution generated by the polishing pad movement mode, the material removal depth at different positions in a specific dwell time is different. Hence, the average removal depth of each polishing point is taken as the total material removal depth $Z$ of the workpiece to achieve an objective consideration of the smoothing effect. $Z$ satisfies the following equation:

$$
Z=\frac{V R R}{\pi R^{2}} \cdot t=K \cdot P \cdot \frac{\omega_{1}}{\pi R^{2}} \int_{0}^{R} r V(r) d r \cdot t
$$

Substituting Eq.3 and Eq.14 into Eq.6, a complete multi-parametric smoothing model can be obtained as follows:

$$
\begin{aligned}
& \varepsilon=\left(\varepsilon_{\text {ini }}-\varepsilon_{0}\right) e^{-\kappa_{\text {total }} \cdot \frac{V R R}{P \cdot \pi R^{2}} \cdot t}+\varepsilon_{0} \\
& =\left(\varepsilon_{\text {ini }}-\varepsilon_{0}\right) e^{-\kappa_{\text {total }} \cdot K \cdot \frac{\omega_{1}}{\pi R^{2}} \int_{0}^{R} r V(r) d r \cdot t}+\varepsilon_{0}
\end{aligned}
$$

An error decreasing factor $(E D F)$ is defined to characterize the efficiency of the exponential convergence over time of the surface ripple errors of the workpiece during the smoothing process, and its equation satisfies:

$$
E D F=\kappa_{\text {total }} \cdot \frac{V R R}{P \cdot \pi R^{2}}=\kappa_{\text {total }} \cdot K \cdot \frac{\omega_{1}}{\pi R^{2}} \int_{0}^{R} r V(r) d r
$$

In this way, the predictable smoothing evolution model Eq.15 with complete parameters of the entire polishing process is simplified as:

$$
\varepsilon=\left(\varepsilon_{i n i}-\varepsilon_{0}\right) e^{-E D F \cdot t}+\varepsilon_{0}
$$

In this model, the surface ripple errors converge exponentially with a certain smoothing efficiency which depends on the magnitude of EDF: larger EDF implies higher efficiency. The convergence curve of the whole smoothing process and then the volume removal rate can be theoretically predicted with the given process parameters. However, due to the 
instability of the pitch layer and the inhomogeneity of the pressure distribution, the actual removal rate might deviate from the theoretic prediction. Therefore, it is necessary to bring in the volume removal efficiency from an actual polishing spot to calculate the EDF based on Eq.16.

\section{Correction of EDF solution process}

According to the parameterized smoothing theory, $\kappa_{\text {total }}$ is com posed of $\kappa_{\text {elastic }}$ and $\kappa_{\text {others }}$. The elastic coefficient $\kappa_{\text {elastic }}$ of the pitch layer is related to the spatial frequency $f$ of the workpiece surface ripple errors [18], while $\kappa_{\text {others }}$ is possibly affected by the geometry of the polishing tool itself, material, polishing slurry, and also the spatial frequency $f$ of ripple errors. Therefore, a parameter $C$, called the slope correction factor, is used instead of $\kappa_{\text {others }}$ [11].

$$
\kappa_{\text {total }}=\frac{1}{\frac{1}{\kappa_{\text {elastic }}(f)}+\frac{1}{C(f)}}
$$

The influence of the spatial frequency $f$ of the ripple errors on the factor $E D F$ will be discussed in the experimental part.

In the parametrized smoothing model, according to Eq.2 and Eq.3, fitting a series of continuous experiment data of the smoothing factor SF and the surface ripple errors as shown in Fig. 3(a), a straight line with fitting slope $k$ as shown in Fig. 3(b) can be obtained. Then $\kappa_{\text {total }}$ can be calculated with $k$ and the pressure $P$ of the polishing pad from Eq.3. At the same time, the convergence curve of the ripple errors in the whole smoothing process can be inferred in accordance with the smoothing model. However, comparing the experimental results, there is a certain difference between the $\kappa_{\text {total }}$ calculated by using the slope $k$ and the $\kappa_{\text {total }}$ obtained by the reverse calculation of the experimental results, which leads to the deviation of the predicted curve and the actual smoothing curve. Hence, it's of vital importance to quantitatively analyze and modify the solution process of the $E D F$ based on the parameterized model in combination with experimental phenomena.

Several short-time pre-processing is usually carried out to achieve the purpose of predicting the smoothing effect, and then the actual smoothing factor $S F$ is calculated by combining these data. As shown in Fig. 3(a), during the pre-polishing process, the surface ripple errors converge exponentially. The actual experimental data is only a series of points on the curve at equal time intervals, denoted as data 1 , data 2, data 3. The linear fit lines corresponding to these data points are shown in the Fig. 3(b). According to the definition of the smoothing factor $S F$ from Eq.2 and Eq.17, for data 1, the following relationship equation between smoothing factor $S F$ and the error decreasing factor $E D F$ can be obtained:

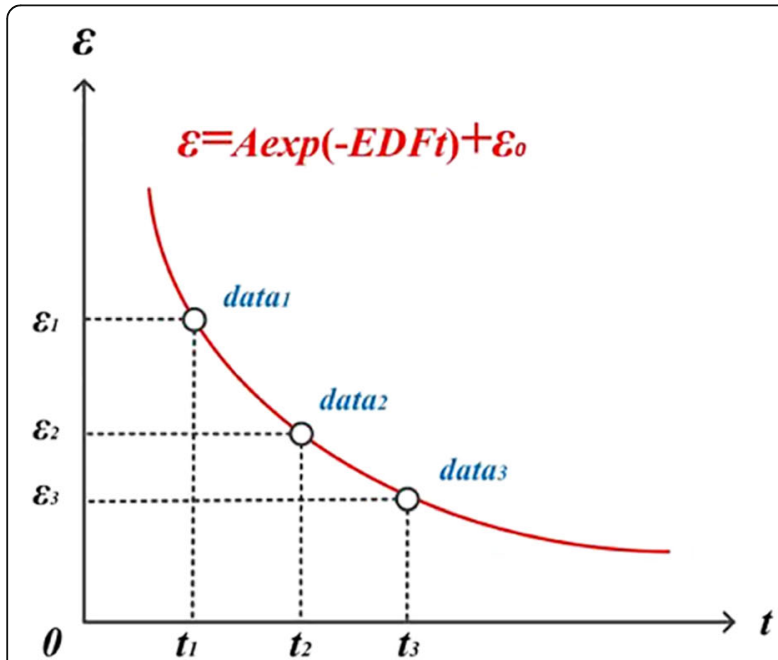

(a)

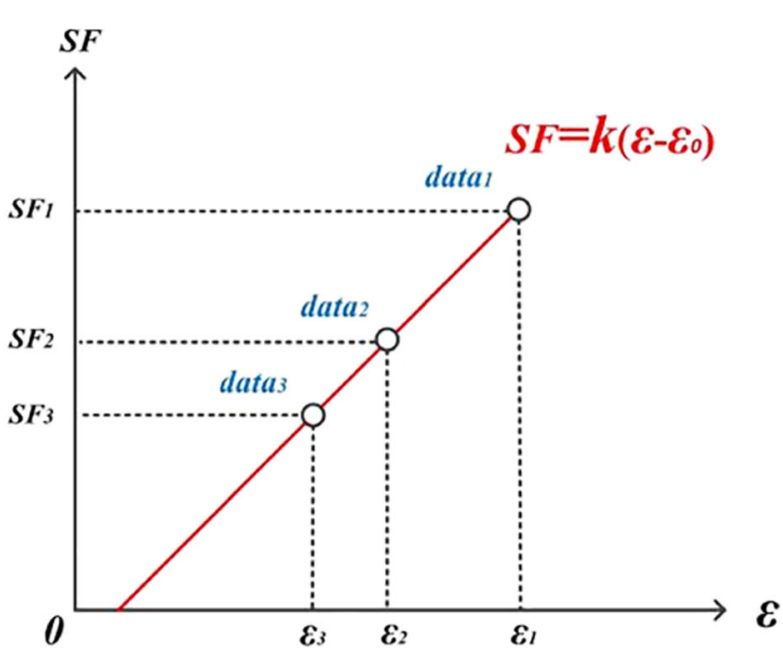

(b)

Fig. 3 a The convergence curve of ripple errors during the actual smoothing process. $\mathbf{b}$ The corresponding data points in the parametric model 


$$
\begin{aligned}
S F_{1} & =\frac{\Delta \varepsilon}{\Delta Z}=\frac{\varepsilon_{1}-\varepsilon_{2}}{\frac{V R R}{\pi R^{2}}\left(t_{2}-t_{1}\right)} \\
& =\frac{A e^{-E D F \cdot t_{1}}-A e^{-E D F \cdot t_{2}}}{\frac{V R R}{\pi R^{2}}\left(t_{2}-t_{1}\right)} \\
& =\frac{A e^{-E D F \cdot t_{1}}\left(1-e^{-E D F \cdot \Delta t}\right)}{\frac{V R R}{\pi R^{2}} \Delta t}
\end{aligned}
$$

Similarly, at the point of data 2, SF satisfies:

$$
\begin{aligned}
S F_{2} & =\frac{A e^{-E D F \cdot t_{2}}\left(1-e^{-E D F \cdot \Delta t}\right)}{\frac{V R R}{\pi R^{2}} \Delta t} \\
= & \frac{A e^{-E D F \cdot t_{1}} e^{-E D F \cdot \Delta t}\left(1-e^{-E D F \cdot \Delta t}\right)}{\frac{V R R}{\pi R^{2}} \Delta t}
\end{aligned}
$$

Then the slope of the line shown in Fig. 3 (b) satisfies:

$$
\begin{aligned}
k_{0} & =\frac{S F_{1}-S F_{2}}{\varepsilon_{1}-\varepsilon_{2}}=\frac{A e^{-E D F \cdot t_{1}}\left(1-e^{-E D F \cdot \Delta t}\right)^{2}}{\frac{V R R}{\pi R^{2}} A e^{-E D F \cdot t_{1}}\left(1-e^{-E D F \cdot \Delta t}\right)} \\
& =\frac{1-e^{-E D F \cdot \Delta t}}{\frac{V R R}{\pi R^{2}} \Delta t}
\end{aligned}
$$

Substituting the expression of EDF in Eq.16 into the above equation gives:

$$
k_{0}=\frac{1-e^{-\frac{\kappa_{\text {total }} \cdot \frac{V R R}{P} \Delta t}{\pi R^{2}} \Delta}}{\frac{V R R}{\pi R^{2}} \Delta t}
$$

It can be seen that under the premise of keeping the process parameters the same, the fitting slope $k_{0}$ will change with the difference of the single pre-processing time $\Delta t$. Affected by this, the $\kappa_{\text {total }}$ obtained by Eq.3 combining the fitting slope $k_{0}$ and the polishing pressure $P$ also becomes a variable affected by the single preprocessing time $\Delta t$, which does not conform to the essence of the processing process. $\kappa_{\text {total }}$ is defined as the material coefficient of the polishing pad, which is usually constant without changing the structure or material of the polishing pad. Therefore, the abnormal experimental phenomena obtained above can be reasonably explained by Eq.22. During the actual polishing process, the relationship between the true value of $\kappa_{\text {total }}$ and the linear slope $k_{O}$ obtained by fitting the parametric smoothing model no longer satisfies Eq.3, which does not mean that Eq.3 is not suitable for the whole smoothing process。The slope $k$ in Eq. 3 is calculated by fitting multiple sets of data points obtained from the complete smoothing process (the ripple errors of the workpiece reach the smoothing limit from the initial amplitude through multiple smoothing processes). Several shortterm smoothing during the pre-processing process, however, causes a certain degree of difference between the fitted slope $k_{0}$ calculated by the fewer data points and the fitting slope $k$ of the complete smoothing process. Under this inducement, the $\kappa_{\text {total }}$ (expressed as $\kappa_{\text {total_(un- }}$ fixed) in the experimental part) and EDF (expressed as $E D F_{-}$(unfixed) in the experimental part) calculated by Eq.3 cannot match the actual values obtained from the smoothing process.

In fact, taking the limit value for the single preprocessing time $\Delta t$, Eq.22 satisfies:

$$
\lim _{\Delta t \rightarrow 0} \frac{1-e^{-\frac{\kappa_{\text {total }} \cdot \frac{V R R}{P} \Delta t}{\pi R^{2}}}}{\frac{V R R}{\pi R^{2}} \Delta t}=\frac{\kappa_{\text {total }}}{P}
$$

Obviously, since the sampling interval $\Delta t$ of the two data points is not zero in the actual polishing, the value of $\kappa_{\text {total }}$ calculated based on the fitted slope $k$ and Eq. 3 is smaller than its actual value. In the case of multiple sampling and ensuring that the sampling interval $\Delta t$ is small enough, the above equation can be satisfied approximately, which is hard to implement in the preprocessing process. Calculating the value of $\kappa_{\text {total }}$ through Eq. 22 can effectively avoid the influence of the sampling interval $\Delta t$ on the solution process of $\kappa_{t o t a l}$ and obtain the true material coefficients normalized by sampling interval and the error decreasing factor of the complete smoothing process. Due to the differences in the structure and characteristics of different polishing pads, the material properties need to be tested after replacing the polishing pad for smooth processing, that is, several short-time pre-processing is required. The real material properties of the current polishing pad can be obtained by substituting the calculated smoothing factor $S F$ and the fitting slope $k_{0}$ into Eq.22, and then the convergence curve of ripple errors in the whole smoothing process can be simulated to realize the prediction and guidance of smoothing processing. At this point, a modified smoothing model has been established completely.

\section{Results and discussions}

Smoothing contrast experiment of ripple errors with different spatial periods

In order to explore the relationship between the error decreasing factor $(E D F)$ and the spatial frequency of ripple errors, a set of smoothing contrast experiments were carried out on three pieces of fused silica (size $100 \mathrm{~mm} \times 100 \mathrm{~mm}$ ) with initial surface error of $3 \mathrm{~mm}$, $5 \mathrm{~mm}$ and $7 \mathrm{~mm}$ obtained through magnetorheological finishing pretreatment, respectively. These three fused silica components were subjected to a continuous 
polishing experiment on the same polishing platform (seen Fig. 1(b)).

The experiment was performed on a pitch pad with a diameter of $35 \mathrm{~mm}$, with eccentricity set to 3 $\mathrm{mm}$, angular velocity of revolution set to $200 \mathrm{rpm}$, of rotation to $20 \mathrm{rpm}$, and the rotation direction being opposite to the revolution direction. On the surface of the component, the polishing pad travels in a grating path at a speed of $200 \mathrm{~mm} / \mathrm{min}$ and a grating spacing of $3 \mathrm{~mm}$. The surface of the three fused silica components was examined initially and after each polishing process with an interferometer. For each fused silica component, the polishing experiment should be stopped when the smoothing effect reaches the limit.

Figure 4 shows the area image measured in the experiment with an error of $7 \mathrm{~mm}$ in space interval. Considering the edge effect in the polishing process and the uniformity of the material removal after the superposition of the TIF, the data taken contains only the area of the original fused silica element with a center diameter of $40 \mathrm{~mm}$, which is about the same size of a TIF scale.

From the experiment, it is clear that the surface ripple errors gradually decrease as the smoothing process goes on. The RMS (root mean square) is reduced from the initial $32.41 \mathrm{~nm}$ to the final $1.57 \mathrm{~nm}$ to reach the smoothing limit, when the mid-spatial frequency errors of the fused silica have been removed. The experimental images of the other two components with ripple errors of $5 \mathrm{~mm}$ and $3 \mathrm{~mm}$ in spatial frequency respectively are similar to the image above. The data collected from the experiment of the three components were analyzed and plotted as shown in Fig. 5, with the RMS value being the ordinate and the number of smoothing times being the abscissa, and an exponential fitting was performed.

The fitting results on the data of $3 \mathrm{~mm}, 5 \mathrm{~mm}$, and 7 $\mathrm{mm}$ spatial spacing are listed as follows:

$$
\begin{aligned}
& R M S_{3 m m}=27.274 e^{-0.630 N}+0.853(\mathrm{~nm}) \\
& R M S_{5 m m}=28.859 e^{-0.471 N}+1.343(\mathrm{~nm}) \\
& R M S_{7 m m}=31.672 e^{-0.183 N}+0.738(\mathrm{~nm})
\end{aligned}
$$

It is found through the experiments that the workpiece with an initial surface error of $3 \mathrm{~mm}$ took the minimum time to reach the smoothing limit, while the workpiece with $7 \mathrm{~mm}$ initial surface error took the longest time. In the experiment, a single smoothing time of each workpiece was set to $17 \mathrm{~min}$, and the average smoothing time of each point in the experimental area was:

$$
\Delta t=\frac{\pi \times 20.5^{2}}{100 \times 100} \times 17 \approx 2 \min
$$

Therefore, the fitted $E D F$ values for the three curves are:

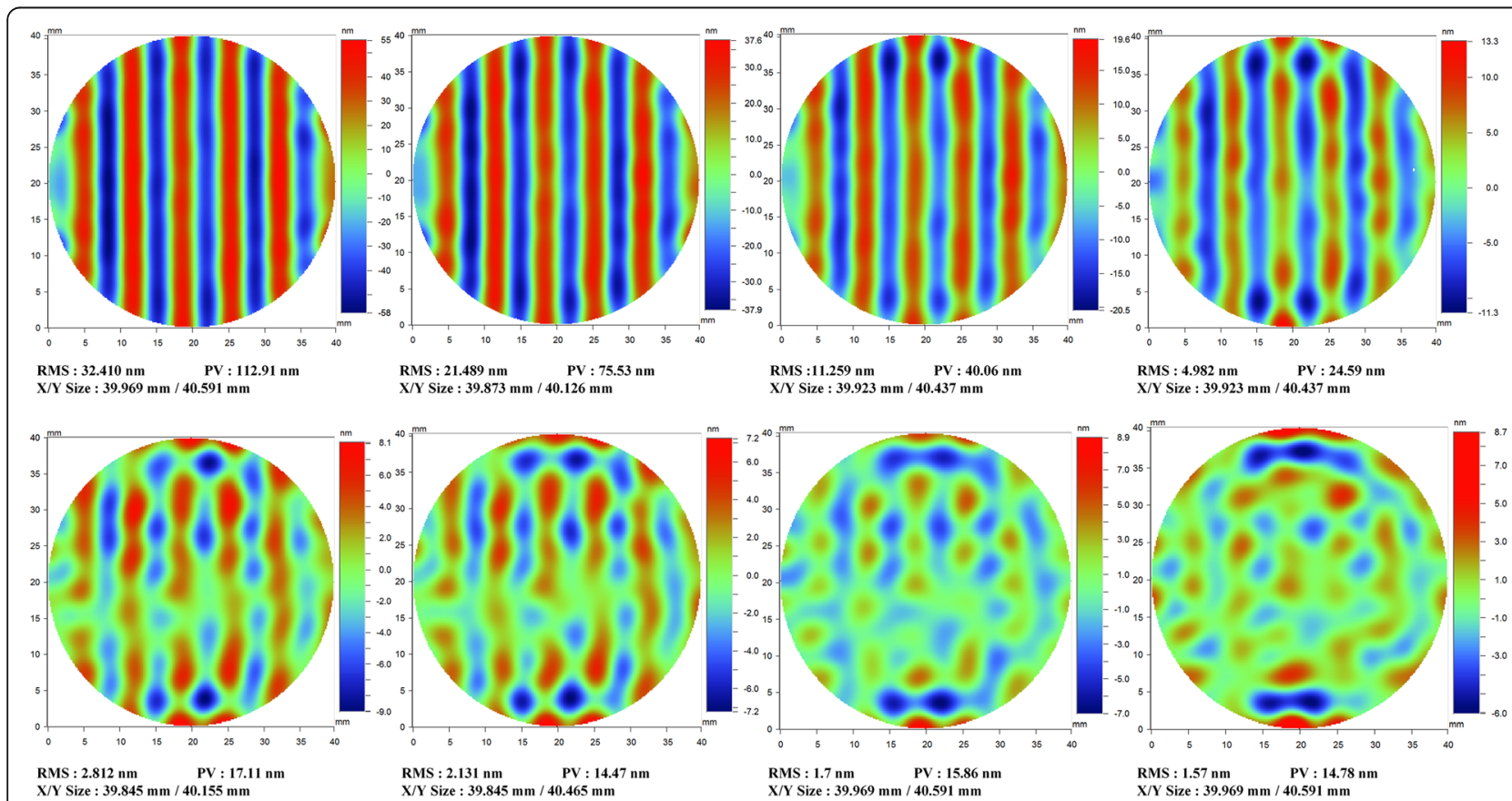

Fig. 4 Surface data of the workpiece surface with a spatial period of $7 \mathrm{~mm}$, which is at the initial time and after the 3rd, 6th, 9th, 12 th, 15 th and the final polishing process 


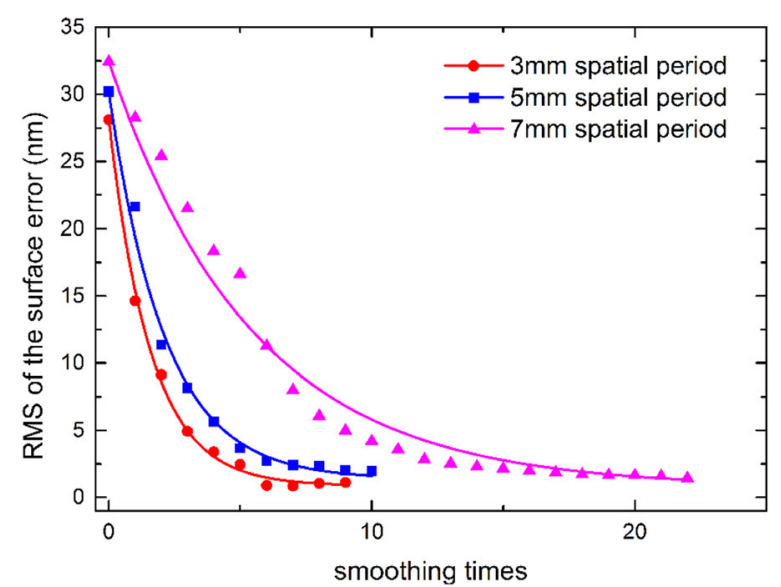

Fig. 5 Data points and the fitting curve of the three components with different spatial period ripple error in the smoothing experiment

$$
\begin{aligned}
& E D F(\text { fitted })_{3 \mathrm{~mm}}=0.310 / \mathrm{min} \\
& E D F(\text { fitted })_{5 \mathrm{~mm}}=0.236 / \mathrm{min} \\
& E D F(\text { fitted })_{7 \mathrm{~mm}}=0.092 / \mathrm{min}
\end{aligned}
$$

In fact, in the three sets of experiments, except for the initial surface ripple error frequency of the workpiece, all other parameters of the polishing process are the same, that is to say, all terms of EDF of the three sets of experiments in the theoretical model are equal except for $\kappa_{\text {total }}$. According to Eq.17, the ripple frequency affects the elastic coefficient $\kappa_{\text {elastic }}(f)$ of the pitch layer and the slope correction factor $C$. Comparing the EDF values of the three sets of experiments, the higher the corrugated space frequency is, the larger the material impact parameter $\kappa_{\text {total }}$ is, which leads to a larger $E D F$. And the larger $E D F$ is, the higher smoothing efficiency is, then the faster the convergence curve of the smoothing experiment decreases.

\section{Experimental verification of the correction effect of parametric smoothing model}

Before the experiment mentioned above, a set of polishing spots were obtained under the same polishing process parameters, as shown in Fig. 6. Due to the inhomogeneous pressure distribution of the actual polishing pad and the instability of the pitch layer, the TIF has a certain deviation from the theory. And in the calculation of the smoothing model, it is the volume removal rate of the actual polishing spot that should be applied, which was $2.06 \times 10^{-2}$ $\mathrm{mm}^{3} / \mathrm{min}$.

According to the parametric smoothing model, a series of data points $(P V)$ of the surface ripple errors on the experimental workpieces and the smoothing factor $(S F)$ were plotted and fitted linearly, as shown in Fig. 7.

The pressure loading during the experiment was always $5 \mathrm{~N}$. With the fitted straight line slope $k_{0}$ shown in Table. 1, using Eq.22, the corresponding $\kappa_{\text {total }}$ value in the experiment can be obtained. Substituting it into the smoothing model Eq.15, together with the parameters of the polishing pad in the experiment, the calculated $E D F$ values corresponding to the spatial frequency can be obtained. In order to verify the correction effect of parametric smoothing model, the original $\kappa_{\text {total }}$ (unfixed) calculated from Eq.3 and the corrected $\kappa_{\text {total }}$ (fixed) calculated according to Eq.22 are listed below. Finally, the error decreasing factors $E D F_{-}$(fitted) fitted to the experimental data were compared, as shown in Table. 1.

$E D F_{- \text {(unfixed) }}$ and $E D F_{- \text {(fixed) }}$ are the $E D F$ values calculated from $\kappa_{\text {total }}$ (unfixed) and $\kappa_{\text {total }}$ (fixed), respectively. From the table, it can be found that the magnitude of the corrected $E D F_{-(\text {fixed) }}$ is closer to the experimental results (with uncertainty within $4.5 \%$ ), while the uncorrected $E D F_{- \text {(unfixed) }}$ has a larger deviation from the actual curve convergence factor (with maximum deviation 38.1\%). Therefore, it can be objectively considered that $\kappa_{\text {total }}$ (fixed) calculated from the modified relationship Eq.22 is closer to the actual data, thereby confirming the accuracy of Eq.22 and the necessity of correction for parametric model.

\section{Prediction of smoothing experiment}

In order to further examine how well the theoretical smoothing model can describe the actual smoothing process, another set of polishing experiment was performed, which is on a round workpiece $(\Phi 150 \mathrm{~mm})$, with initial surface ripple spital interval set to $5 \mathrm{~mm}$. A polishing pad with a diameter of $50 \mathrm{~mm}$ and eccentricity of 5 $\mathrm{mm}$ were chosen in the progress. Different from the previous experiments, the volume removal rate measured through the polishing spot before smoothing experiment was $V R R=1.445 \times 10^{-1} \mathrm{~mm}^{3} / \mathrm{min}$, and the pressure given during polishing was $55 \mathrm{~N}$. The experimental result is shown in Fig. 8.

In Fig. 8, the red curve is the predicted curve calculated by the theoretical parameterized model. Since the material of the polishing pad selected in the experiment was the same as the previous experiment, and the spital interval of the surface ripple errors is $5 \mathrm{~mm}$, the value of $\kappa_{\text {total }}$ used in the theoretical prediction is $77.357 /$ $\left(\mathrm{pa} \cdot \mathrm{nm}^{-1}\right)$ as listed in Table.1. According to the parametric smoothing model, the theoretical error decreasing factor $E D F_{2}$ calculated by Eq.15 satisfies: 


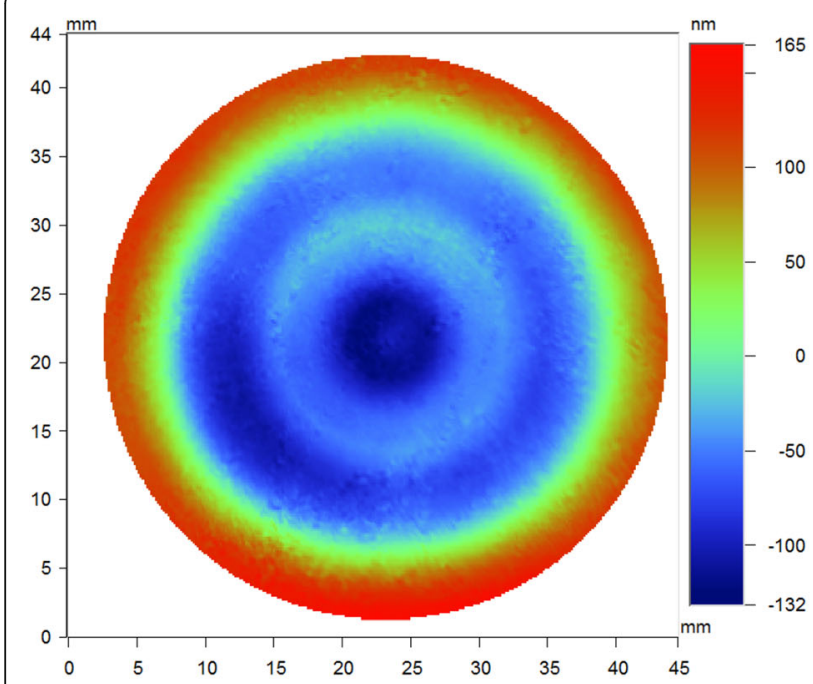

(a)

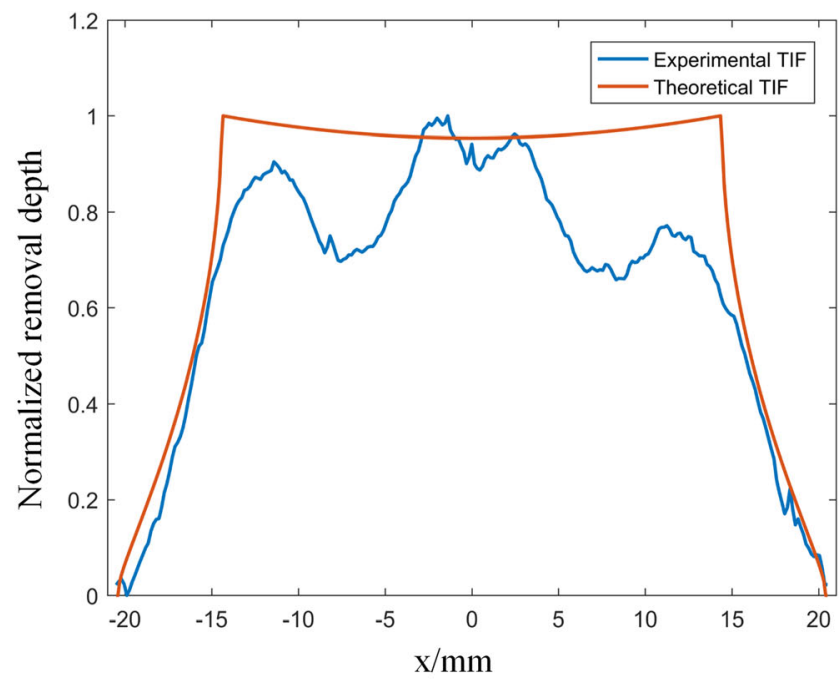

(b)

Fig. 6 Polishing spot of the experience and the normalized TIF

$$
\begin{aligned}
E D F_{2}= & \frac{77.357 \mathrm{pa} \cdot \mathrm{nm}^{-1}}{\frac{55 \mathrm{~N}}{\pi \times 25^{2} \mathrm{~mm}^{2}}} \\
& \times \frac{1.445 \times 10^{-1} \mathrm{~mm}^{3} \cdot \mathrm{min}^{-1}}{\pi \times 30^{2} \mathrm{~mm}^{2}} \\
= & 0.141 \mathrm{~min}^{-1}
\end{aligned}
$$

Similarly, the actual smoothing time of each point in the polishing area is $3 \mathrm{~min}$, so the actual $E D F_{2}$ (fitted) obtained from the fitting curve of the experimental data is:

$$
E D F_{2}(\text { fitted })=0.433 \div 3=0.144 \mathrm{~min}^{-1}
$$

It shows that there is a better match between the smoothing convergence curve obtained by theoretical calculation and the experimental curve within a certain

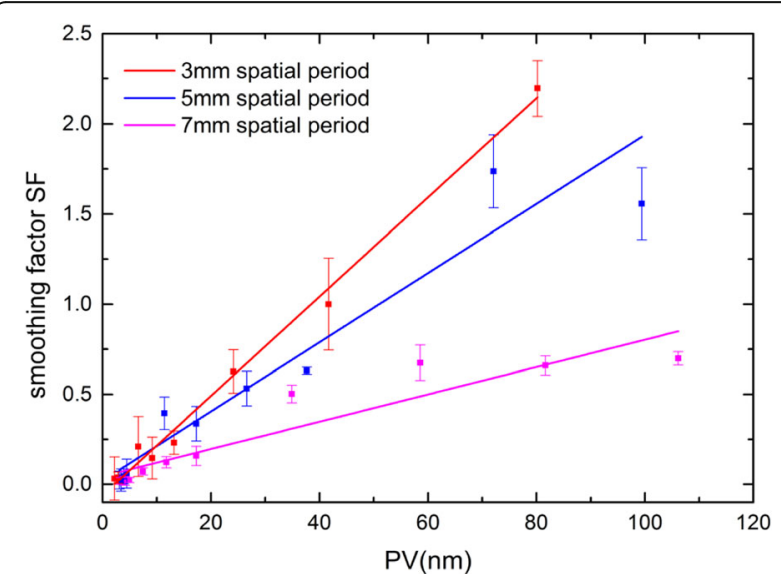

Fig. 7 Linear fitting of the smoothing factor and the surface ripple errors. In the experiment, smoothing time of each workpiece was $17 \mathrm{~min}$ uncertainty. In this experiment, the deviation of each data point from the theoretical prediction does not exceed $2 \%$.

The source of the uncertainties is from the fitted slope $k_{0}$ and the instability of the experimental conditions: the slope $k_{0}$ obtained from the linear fit on the experimental data is not accurate, therefore propagating to calculating $\kappa_{\text {total }}$. At the same time, there is still a certain difference between the polishing pads of the two groups due to the instability of the pitch layer and the change in thickness, which can affect the actual material coefficient. In general, the theoretical model predicts the experimental results from numerical calculations well, and gives a certain accuracy of the predicted curve for the actual processing, application and optimization of the selection of polished spots to smooth the surface ripple errors.

\section{Conclusion}

Based on the urgent need for quantitative prediction of smoothing effect, a new predictable smoothing evolution model for computer-controlled polishing was established. The main focus of this study was its basic smoothing

Table. 1 Parameters calculated results

\begin{tabular}{llll}
\hline Spatial period & $\mathbf{3} \mathbf{~ m m}$ & $\mathbf{5 ~} \mathbf{~ m}$ & $\mathbf{7 ~} \mathbf{~ m m}$ \\
\hline$k_{0} / \mathrm{nm}^{-1}$ & 0.0153 & 0.0097 & 0.0044 \\
$K_{\text {totaL_(unfixed) }} / \mathrm{pa} \mathrm{nm}^{-1}$ & 79.553 & 48.876 & 22.878 \\
$K_{\text {totaL(fixed) }} / \mathrm{pa} \mathrm{nm}^{-1}$ & 108.045 & 77.357 & 29.465 \\
$E D F_{\text {(unfixed) }} / \mathrm{min}^{-1}$ & 0.238 & 0.146 & 0.069 \\
$E D F_{\text {(fixed) }} / \mathrm{min}^{-1}$ & 0.324 & 0.231 & 0.088 \\
$E D F_{\text {-(fitted) }} / \mathrm{min}^{-1}$ & 0.310 & 0.236 & 0.092 \\
\hline
\end{tabular}




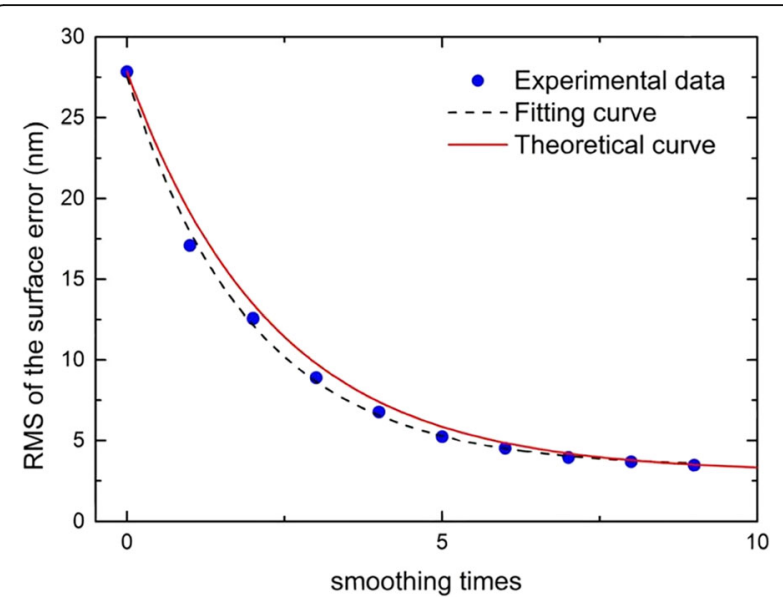

Fig. 8 The comparison of predicted results and experimental results

theory, solution method and modification. Firstly, combining the existing qualitative characterization equation of the convergence process of the ripple errors, the parametric smoothing model containing time variables suitable for computer-controlled polishing was proposed. An error decreasing factor $E D F$ was defined, as an integral factor that contains the various process parameters of the polishing process, which characterizes the smoothing efficiency under some specific settings of polishing process. According to the several data points and process parameters obtained by the short-time pre-processing before smoothing, the EDF and theoretical error convergence curve of the current smoothing process can be calculated.

In view of the observed experimental phenomenon that the theoretical predicted curve deviates from the actual curve, the solution process of the parameterized smoothing model was revised and verified by experiments. After correction, the maximum deviation of the EDF obtained by theoretical calculation and the EDF obtained by experimental fitting is reduced from $38.1 \%$ to $4.5 \%$. On this basis, the predicted experiment for the actual complete smoothing process was carried out by using the parametric model, which indicated that the predicted curve is in good agreement with the actual smoothing curve and the deviation between theoretical data points and actual values is not more than $2 \%$.

The error decreasing factor EDF specifically includes the polishing pad geometry, rotational speed, material, and physicochemical environmental factors during the polishing process, and the spatial frequency of the ripple errors. It can be concluded from the smoothing experiments that the spatial frequency of the surface ripple error does have an effect on the smoothing efficiency. In the same smoothing environment, the smoothing efficiency of the ripple errors with a larger spatial frequency is higher, and the convergence curve falls faster.
The predictable smoothing evolution model proposed in this paper shows high accuracy and good universality, so that the research on the smoothing effect of ripple errors in computer-controlled polishing is no longer limited to qualitative analysis but can achieve quantitative prediction. In addition, it also provides a certain degree of theoretical support and guidance for the adjustment of the process conditions of smoothing the ripple errors.

\section{Abbreviations}

MSFE: Mid-spatial frequency error; CCOS: Computer-controlled optical surfacing; EDF: Error decreasing factor; SF: Slip factor; TIF: Tool influence function; VRR: Volume removal rate

\section{Acknowledgements}

Not applicable.

\section{Authors' contributions}

Jing Hou and Pengli Lei put forward the predictable evolution smoothing model, Shiwei Liu modified the solution of $K_{\text {total, }}$ and Xianhua Chen and others assisted in the verification of the smoothing model and the verification of the smoothing effect of ripples of different periods. The author(s) read and approved the final manuscript.

\section{Funding}

This work was financially supported by the Science Challenge Project (grant numbers: No.TZ2016006-0502-01) and High-grade CNC Machine Tool and Basic Manufacturing Equipment Project (grant numbers: No.

2017ZX04022001-101).

Availability of data and materials

Data will be shared after publication.

Ethics approval and consent to participate

Not applicable.

Consent for publication

Not applicable.

Competing interests

The authors have declared that no competing interests exist.

\section{Author details}

${ }^{1}$ Research Center of Laser Fusion, China Academy of Engineering Physics, Mianyang 621900, China. ${ }^{2}$ School of Mechatronics \& Engineering, Harbin Institute of Technology, Harbin 150001, China.

Received: 8 September 2019 Accepted: 16 November 2020

Published online: 25 November 2020

\section{References}

1. Jones, R.A.: Optimization of computer-controlled polishing [J]. Appl. Opt. 16(1), 218-224 (1977)

2. Nelson, J., Sanders, G.H.: The status of the thirty meter telescope project [J]. Ground-based. Airborne Telescopes II. 7012, 70121A (2008)

3. Johns, M., McCarthy, P., Raybould, K.: Giant Magellan telescope: overview [J]. Ground-based. Airborne Telescopes IV. 8444, 84441H (2012)

4. Wagner, R.E., Shannon, R.R.: Fabrication of aspherics using a mathematical model for material removal [J]. Appl. Opt. 13(7), 1683-1689 (1974)

5. Wang, Y., Ni, Y., Yu, J.: Computer-controlled polishing technology for small aspheric lens [J]. Opt. Precis. Eng. 15(10), 1527-1533 (2007)

6. Wang, C., Wang, Z., Yang, X., et al.: Modeling of the static tool influence function of bonnet polishing based on FEA [J]. Int. J. Adv. Manuf. Technol. 74(1-4), 341-349 (2014)

7. Dong, Z., Cheng, H., Tam, H.: Modified subaperture tool influence functions of a flat-pitch polisher with reverse-calculated material removal rate [J]. Appl. Opt. 53(11), 2455-2464 (2014) 
8. Brown, N.J., Baker, P.C., Parks, R.E.: Polishing-to-figuring transition in turned [J]. Optics Contemporary Methods of Optical Fabrication. 306, 58-66 (1982)

9. Mehta, P.K., Reid, P.B.: Mathematical model for optical smoothing prediction of high-spatial-frequency surface errors [J]. Optomechanical Engineering and Vibration Control. 3786, 447-460 (1999)

10. Mehta PK, Hufnagel RE:: Pressure distribution under flexible polishing tools: I. Conventional aspheric optics [J]. Advanc. Opt.Structure. Syst. 1303, 178-88 (1990)

11. Kim, D.W., Park, W.H., An, H.K., Burge, J.H.: Parametric smoothing model for visco-elastic polishing tools [J]. Opt. Express. 18(21), 22515-22526 (2010)

12. Kim, D.W., Martin, H., Burge, J.H.: Control of mid-spatial-frequency errors for large steep aspheric surfaces [J]. Optical Fabrication and Testing. OM4DOM41D (2012)

13. Shu, Y., Nie, X., Shi, F., Li, S.: Smoothing evolution model for computer controlled optical surfacing [J]. J. Opt. Technol. 81(3), 164-167 (2014)

14. Dunn, C.R., Walker, D.D.: Pseudo-random tool paths for CNC sub-aperture polishing and other applications [J]. Opt. Express. 16(23), 18942-18949 (2008)

15. Tam, H.Y., Cheng, H., Dong, Z.: Peano-like paths for subaperture polishing of optical aspherical surfaces [J]. Appl. Opt. 52(15), 3624-3636 (2013)

16. Nie, X., Li, S., Shi, F., Hu, H.: Generalized numerical pressure distribution model for smoothing polishing of irregular mid-spatial frequency errors [J]. Appl. Opt. 53(6), 1020-1027 (2014)

17. Zhang, Y., Wei, C., Shao, J., et al.: Correction of mid-spatial-frequency errors by smoothing in spin motion for CCOS []]. Optical Manufacturing and Testing XI. 9575, 95750D (2015)

18. Kim, D.W., Burge, J.H.: Rigid conformal polishing tool using non-linear viscoelastic effect [j]. Opt. Express. 18(3), 2242-2257 (2010)

19. Nie, X., Li, S., Hu, H., Li, Q.: Control of mid-spatial frequency errors considering the pad groove feature in smoothing polishing process [J]. Appl. Opt. 53(28), 6332-6339 (2014)

\section{Publisher's Note}

Springer Nature remains neutral with regard to jurisdictional claims in published maps and institutional affiliations.

\section{Submit your manuscript to a SpringerOpen ${ }^{\circ}$ journal and benefit from:}

- Convenient online submission

- Rigorous peer review

- Open access: articles freely available online

High visibility within the field

- Retaining the copyright to your article

Submit your next manuscript at $\boldsymbol{\nabla}$ springeropen.com 